computed tomography demonstrated extensive small airway plugging with a "tree-in-bud" pattern associated with early lower lobe bronchiectasis (fig. 1). A clinical diagnosis of DPB was made and he commenced $500 \mathrm{mg}$ of erythromycin twice daily. Over the course of the following 6 months, he experienced a dramatic improvement; his productive cough and rhinnorhoea resolved completely, exercise tolerance returned to normal, and lung field infiltrates on plain chest radiograph resolved while his FEV1 and FVC improved to supra-predicted values (fig. 2). During this period, his ICS/ LABA therapy was not altered. Over the following period the erythromycin dose was reduced to $250 \mathrm{mg}$ twice daily, but within a few weeks he had experienced a recurrence of symptoms and a small, but persistent, drop in FEV1. This improved on increasing the erythromycin dose to $500 \mathrm{mg}$ twice daily, which he remains on at the present time. Although the patient did not undergo surgical lung biopsy, the clinicoradiological presentation and response to macrolide therapy is highly consistent with a diagnosis of DPB.

This case highlights the importance of considering this rare illness in patients of non-Asian origin who present with difficult airways disease. Without appropriate macrolide

\section{Cannabis and lung cancer}

\section{To the Editors:}

The elegant and eminent study by ALDINGTON et al. [1] of the Cannabis and Respiratory Disease Research group is to be welcomed at a time when, as the authors rightly point out, there are many suggestive case studies and clinical series on lung cancer, and indeed other tumours, occurring particularly at younger ages in past and present smokers of cannabis. The authors' careful methodology and succinct summary of much of the relevant literature in relation to this subject is a relevant and timely addition to the literature, particularly in the context of increased interest in this subject in both lay and professional circles.

The point made by ALDINGTON et al. [1] in relation to the lack of research into the molecular underpinnings of cannabis-related oncogenesis is particularly relevant at a period in research history when the molecular bases of disease have received unprecedented attention. It is to be expected that their elucidation might lead to better understanding of the mechanisms of common disorders including cancer and, in time, improved therapeutics. Interestingly, cannabis use also featured in our case review of malignant and pre-malignant disorders of the cervix uteri in an addicted population [2].

From the vast published literature on cannabis, several main pathways emerge as being most relevant to oncogenesis in addition to the obvious factors related to carcinogen content and smoking technique. Cancer is defined as a disorder of uncontrolled cell growth, and mechanisms of DNA toxicity and therapy, the patient faces the prospect of a disease characterised by inexorable decline and early death; however, with appropriate therapy the patient can expect a far better prognosis and quality of life.

\section{N.P. Adams and J. Congelton}

Dept of Respiratory Medicine, Worthing Hospital, Worthing, UK.

\section{STATEMENT OF INTEREST}

None declared.

\section{REFERENCES}

1 McGrath EE, McLaughlin AM, Fitzgerald MX. Diffuse panbronchiolitis: East meets West. Eur Respir J 2007; 29: 817-818.

2 Poletti V, Casoni G, Chilosi M, Zompatori M. Diffuse panbronchiolitis. Eur Respir J 2006; 28: 862-871.

DOI: $10.1183 / 09031936.00011408$ dysregulated DNA replication are its genetic hallmarks. Damage to DNA, therefore, as might occur with free oxygenand nitrogen-centred radicals, is relevant to molecular pathogenesis. Indeed, normal signalling of the endogenous cannabinoid ligand, anandamide, via the oxidative damage of guanine to 6-oxoguanine and its routine restoration by base excision repair has been described in cultured cells [3]. Free radical generation both at receptor binding (S.T. Carney, North Carolina Central University, Durham, NC, USA; personal communication) and by mitochondrial uncoupling [4] has been demonstrated.

Cannabis is widely acknowledged to stimulate the mitogen activated protein kinase (MAPK) pathway, which is a major stimulant of developmental and malignant cell growth. MAPK dysregulation has been identified clinically in tuberous sclerosis, neurofibromatosis and acute myelomonocytic leukaemia (AMML), and greatly increased incidences of AMML have been identified in paediatric populations after in utero maternal exposure to cannabis [5]. There are reports in subacutely "stoned" animals (a use pattern reminiscent of that seen in many patients) that heavy cannabis use is associated with severe telomeric (end-chromosomal) damage in male germ cells [6]. The burgeoning literature on telomeres demonstrates that this field is intimately involved in pathways of both ageing and tumourigenesis. Key DNA repair enzymes topoisomerase II [7] and Rad51 have been shown to be inhibited by cannabinoids. Germ line chromosomal abnormalities in addition to MAPK stimulation constitute putative pathways of inheritable oncogenesis. 
Reports that cannabis is associated with severe dental disease [8], osteoporosis [9] and mental illness [10], together with unpublished studies from our clinic showing accelerated vascular ageing in heavy cannabis smokers, suggest that cannabis may be modifying the ageing process itself. Ageing is of course the leading risk factor for most nonpaediatric tumours. It is well known that there is extensive cross-talk including heterodimerisation at the cell membrane between the cannabinoid type 1 receptor and other guanosine triphosphate-coupled receptors including adrenergic receptors which are well known to be involved in ageing processes. Cannabinoids are also known to induce cell cycle arrest, senescence and apoptotic cell programmes. Senescence is increasingly associated with oncogenic induction [11]. Cannabis has well-recognised immunosuppressive properties [12] that are likely to be of relevance to tumour surveillance activities of the immune system, both in the pre-clinical stages and in the metastatic spread of advanced disease.

Sleep disturbances are clinically prominent and highly problematic in cannabis-dependent patients and imply significant disruption to the cellular circadian genetic clock mechanisms [13]. Circadian disruption has been linked to acceleration of the ageing process in mammals [14]. Finally, it is not widely appreciated that histone deacetylases, central regulators of the epigenetic code, have been shown to be major regulators of ageing [15] (including neurogenesis [16]), psychopathology [17], addiction [16] and cancer [18].

In summary, while cannabinoids, and especially smoked inhaled cannabis, are strongly implicated in oncogenesis by several molecular pathways, there are multiple leads that might prove useful for mechanistic exploration and perhaps, one day, therapeutic exploitation of the cannabinoid system.

\section{A.S. Reece}

University of Queensland Medical School, Highgate Hill, Brisbane, Australia.

\section{STATEMENT OF INTEREST}

None declared.

\section{REFERENCES}

1 Aldington S, Harwood M, Cox B, et al. Cannabis use and risk of lung cancer: a case-control study. Eur Respir J 2008; 31: $280-286$.

2 Reece A. Lifetime prevalence of cervical neoplasia in addicted and medical patients. Aust NZ J Obstet Gynaecol 2007; 47: 419-423.
3 Sarker KP, Obara S, Nakata M, Kitajima I, Maruyama I. Anandamide induces apoptosis of PC-12 cells: involvement of superoxide and caspase-3. FEBS Lett 2000; 472: 39-44.

4 Sarafian TA, Habib N, Oldham M, et al. Inhaled marijuana smoke disrupts mitochondrial energetics in pulmonary epithelial cells in vivo. Am J Physiol Lung Cell Mol Physiol 2006; 290: L1202-L1209.

5 Robison LL, Buckley JD, Daigle AE, et al. Maternal drug use and risk of childhood nonlymphoblastic leukemia among offspring. An epidemiologic investigation implicating marijuana (a report from the Childrens Cancer Study Group). Cancer 1989; 63: 1904-1911.

6 Zimmerman AM, Zimmerman S, Raj AY. Effects of cannabinoids on spermatogenesis in mice. In: Nahas GG, Sutin KM, Harvey DJ, Agurell S, eds. Marihuana and Medicine. Totawa, Humana Press, 1999; pp. 347-358.

7 Kogan NM, Schlesinger M, Priel E, et al. HU-331, a novel cannabinoid-based anticancer topoisomerase II inhibitor. Mol Cancer Ther 2007; 6: 173-183.

8 Hujoel PP. Destructive periodontal disease and tobacco and cannabis smoking. JAMA 2008; 299: 574-575.

9 Idris AI, van 't Hof RJ, Greig IR, et al. Regulation of bone mass, bone loss and osteoclast activity by cannabinoid receptors. Nat Med 2005; 11: 774-779.

10 Moore TH, Zammit S, Lingford-Hughes A, et al. Cannabis use and risk of psychotic or affective mental health outcomes: a systematic review. Lancet 2007; 370: 319-328.

11 Campisi J. Senescent cells, tumor suppression, and organismal aging: good citizens, bad neighbors. Cell 2005; 120: 513-522.

12 Cabral GA. Drugs of abuse, immune modulation, and AIDS. J Neuroimmune Pharmacol 2006; 1: 280-295.

13 O'Leary DS, Block RI, Turner BM, et al. Marijuana alters the human cerebellar clock. Neuroreport 2003; 14: 1145-1151.

14 Kondratov RV, Kondratova AA, Gorbacheva VY, Vykhovanets OV, Antoch MP. Early aging and age-related pathologies in mice deficient in BMAL1, the core component of the circadian clock. Genes Dev 2006; 20: 1868-1873.

15 Guarente L, Picard F. Calorie restriction - the SIR2 connection. Cell 2005; 120: 473-482.

16 Renthal W, Maze I, Krishnan V, et al. Histone deacetylase 5 epigenetically controls behavioral adaptations to chronic emotional stimuli. Neuron 2007; 56: 517-529.

17 Tsankova N, Renthal W, Kumar A, Nestler EJ. Epigenetic regulation in psychiatric disorders. Nat Rev Neurosci 2007; 8: 355-367.

18 Egger G, Liang G, Aparicio A, Jones PA. Epigenetics in human disease and prospects for epigenetic therapy. Nature 2004; 429: 457-463. 